\title{
Identifying the "Active Ingredients" of a School-Based, Workplace Safety and Health Training Intervention
}

\author{
Mikko Nykänen ${ }^{1} \odot \cdot$ Rebecca J. Guerin ${ }^{2} \cdot$ Jukka Vuori $^{1}$
}

Accepted: 12 January 2021 / Published online: 22 January 2021

(c) The Author(s) 2021

\begin{abstract}
Young workers in many industrialized countries experience a higher rate of largely preventable occupation-related injuries compared with adults. Safety education and training are considered critical to the prevention of these incidents. This can be promoted by the dissemination and scale-out of an evidence-based, safety training programs in vocational education. The aim of this study was to identify the intervention core components that comprise the "active ingredients" of a safety training intervention for young workers and assess the impact on student outcomes of interest. Fidelity of implementation was operationalized using measures of adherence and quality of intervention delivery. For this study, data were collected through a school-based, cluster randomized trial conducted in 2015 in eight Finnish upper secondary-level vocational schools $(n=229$ students in 22 groups, each with one teacher). Results indicate that the intervention core components (safety skills training, safety inoculation training, a positive atmosphere for safety learning, and active learning techniques) had differing associations with student outcomes. Adherence related to the acquisition of safety skills training was the strongest active ingredient in terms of positive effects. Furthermore, quality of delivery in terms of fostering positive learning atmosphere and utilizing active learning methods was associated especially with motivational outcomes. These findings indicate that different active ingredients complemented each other. Contrary to expectations, we found no statistically significant relationship between any of the core components and risk-taking attitudes. The current study advances prevention science by identifying the active ingredients of an evidence-based intervention, implemented in Finnish vocational school settings, that helps protect young workers from work-related morbidity and mortality.
\end{abstract}

Keywords Young worker · Occupational safety and health · Fidelity of implementation · Vocational education · Injury prevention

\section{Introduction}

Previous research indicates that younger workers (defined as adolescents and young adults aged under 30 years) are at an elevated risk of being injured (and in some cases fatally) at work (Breslin \& Smith, 2006; Hanvold et al., 2019; Guerin et al., 2020). Inexperience, exposure to job hazards and unsafe tasks (such as heavy lifting), psychosocial factors (such as low job control), and organizational factors (such

Mikko Nykänen

mikko.nykanen@ttl.fi

1 Finnish Institute of Occupational Health, PO Box 40, 00032 Helsinki, Finland

2 Division of Science Integration, National Institute for Occupational Safety and Health, US Centers for Disease Control and Prevention, Cincinnati, OH, USA as poor safety climate) are just some of the multiple factors associated with increased injury risk for young workers (Breslin \& Smith, 2006; Hanvold et al., 2019). These incidents have been shown to have long-term, negative impacts on adolescent/young adult health and development (Koehoorn et al., 2008). Vocational education plays an important role in preparing young people with knowledge and skills for safe and healthy work (Boini et al., 2017; Rodrigues et al., 2018). Guerin et al., (2020) highlighted that psychological theories provide guidance for developing school-based interventions to prepare for young people for hazards they may face in the workplace. However, it is noteworthy that there have been relatively few schoolbased OSH intervention studies based on psychological theories. According to a previous randomized-controlled trial (Nykänen et al., 2018, 2019), a safety training approach based on social-cognitive framework (Bandura, 
1997; Rotter, 1982) and expectancy-value theory (Eccles $\&$ Wigfield, 2002) had positive outcomes among students in vocational education. However, there is need for a more thorough understanding of the processes underlying intervention efficacy and effectiveness and to identify which aspects, or components, of the intervention are the main contributors to the outcomes of interest.

Previous research has emphasized the importance of core components, derived from theory or empirical evidence, that are most likely to account for the main effects of successful interventions (Backer, 2001; Fixsen et al., 2009; Irwin \& Supplee, 2012). Not all core components are created equal, with some linked to stronger intervention effects more than others (Abry et al. 2015). When the most important core components of an intervention are identified, efforts to replicate or adapt that program will be more successful because these key elements can be kept intact (Espada et al., 2012). How to identify what is essential is an important challenge in the successful implementation of evidence-based programs (Durlak \& DuPre, 2008) and can be facilitated by the use of theoretical frameworks during intervention development (Allen et al., 2018). Given that the core components represent the program theory/internal logic, fidelity entails that these essential elements are implemented in the manner intended by program developers (Allen et al., 2018; Dusenbury et al., 2003; Mowbray et al., 2003). Said differently, intervention core components are the program elements hypothesized to transmit effects and therefore are the target of fidelity assessments (Abry et al., 2015).

The increased attention in prevention research on the measurement of fidelity is evidenced by the proliferation of fidelity models and measurement approaches (e.g., Berkel et al., 2011; Carroll, 2020; Dane \& Schneider, 1998; Gearing et al., 2011; Schoenwald et al., 2011). Although none are definitive (Berkel et al., 2011), two common features of most fidelity frameworks and models advanced by researchers include (1) how much and (2) how well a practice was used as intended (i.e., dose or amount of program delivered, adherence to the program protocol, quality of program delivery, and participant acceptance). (Dunst et al., 2013; Rohrbach et al., 2006). Efforts to test the hypothesized, essential elements of interventions are rare (Durlak \& DuPre, 2008; Abry et al., 2015). Research is needed on how to identify, through empirical investigation, the so-called "active ingredients" of interventions and explore which are most responsible for improving program outcomes (Abry et al., 2015; Collins et al., 2005; Durlak $\&$ Dupre, 2008). More research in this area has also been called for in the occupational safety and health (OSH) field (Dugan \& Punnett, 2017; Schulte et al., 2017). In sum, knowledge of a program's active ingredients can be used to identify specific practices, when implemented with fidelity, that facilitate desired change in participants, to optimize intervention effectiveness, and to reduce costs and burden on program participants and implementers (Abry et al., 2015).

To address a gap in the prevention science, public health, and OSH research, we explored the associations between the hypothesized core components, implemented with fidelity, and intervention outcomes of a school-based, OSH training program, Attitude to Work, developed in 2015 by the Finnish Institute of Occupational Health (FIOH), to identify active intervention ingredients.

\section{Attitude to Work Program}

Attitude to Work is a safety training program targeted at students in upper secondary level vocational education. The intervention was developed in collaboration with vocational schools and workplaces. The program consists of two full, consecutive training days $(12 \mathrm{~h})$ and is implemented by teachers. The program is publicly available online and includes a practical, easy-to-use facilitator's guide with ready-to-use lesson plans and concrete examples of safety training activities. Train-the-trainer workshops conducted by FIOH provide pedagogical tools, resources, and professional development support for teachers. The program is highly structured, providing detailed instructional guidelines for the teacher to implement the program with a student group. The teachers also receive a two-day training session provided by FIOH before implementing the program.

The intervention mechanism is based on social cognitive theory (Bandura, 1997; Rotter, 1982) and expectancy-value theory (Eccles \& Wigfield, 2002). These two theories share many similarities by addressing the role of competencerelated beliefs in human behavior, but they also complement one another (see Leaper, 2011). Competence-related beliefs such as self-efficacy have received more emphasis in social cognitive theory, whereas the expectancy-value theory outlines competence related beliefs, perceived cost, and utility as important behavioral determinants. Perceived cost refers to negative aspects of engaging in the behavior, and perceived utility refers to the usefulness of the activity to persons' future. (Leaper, 2011; Bandura, 1997; Eccles \& Wigfield, 2002). Hence, a combination of these theories may offer a more comprehensive framework for influencing antecedents of human safety behavior and provide guidance for developing educational safety interventions. In line with the key aspects of social-cognitive theory and expectancy theory, the Attitude to Work safety training program stresses personal control over safety, focuses on competencerelated beliefs, and guides students to identify the positive 
outcomes of preventive actions and negative consequences of risk-taking. Moreover, Attitude to Work promotes a pedagogical approach to safety learning that includes active participation on the part of learners (e.g., modeling, feedback, reflection and dialogue) (see Burke et al., 2006).

In the current study, we assessed the Attitude to Work program in terms of four core components that are hypothesized to represent the underlying intervention mechanism. Fidelity thus requires that these core elements are implemented, as designed, during program delivery. Major features of the intervention are based on its (a) educational content that focuses on safety skills training, (b) educational content that focuses on safety inoculation training, (c) utilizing active learning methods, and (d) fostering positive atmosphere for safety learning. These core components represent the internal logic of the intervention and can be measured to identify intervention program active ingredients serving as key levers of change (Abry et al., 2015). Next, we provide a description of the core components of Attitude to Work training program.

Safety Skills Training Safety skills training involves identifying hazards at the workplace, analyzing the factors that precede incidents and the relationship between unsafe behavior and work-related morbidity and mortality, identifying behavioral strategies for preventing injuries and illnesses, learning about the negative consequences of staying silent about safety issues and the positive consequences of information-seeking and speaking about safety at work, and setting personal, occupational safety goals. During the safety skills training, students are guided toward identifying controllable causes of work-related morbidity and mortality and recognizing personal control over safety.

Safety Inoculation Training This core component is related to practicing how to act when encountering coworkers' risky behavior at the workplace, unfamiliar work tasks, or unsafe work situations. The key idea in safety inoculation training is that the students are guided to identify behavioral strategies to overcome barriers to safe work. Students reflect on possible solutions to challenges and how to implement them in practice. Students are guided to acknowledge their own personal opportunities and means of overcoming challenges that undermine safe work.

Fostering a Positive Atmosphere for Safety Learning The third intervention component promotes safety learning through emotional motivation and fostering active and engaging learning environments. This intervention component supports peer reinforcement during training activities and facilitates emotional motivation in safety learning. Intervention guidelines provide teacher instructions on how to foster a supportive, respectful, and engaging learning environment during program implementation.
Use of Active Learning Techniques Instructional techniques are based on the learner's own active participation. The program promotes interaction and dialogue between students as they share their safety-related experiences, knowledge, and skills. The intervention program includes group discussions, role play and small-group tasks, and problem-solving exercises. Instead of lecturing, the trainers use the knowledge, ideas, and experiences of the participants themselves as part of the learning process.

\section{Earlier Findings on Intervention Impact}

Results from previous research (Nykänen et al., 2018, 2019) indicate that Attitude to Work had beneficial outcomes in terms of safety preparedness, the internal safety locus of control, risk attitudes, and the safety motivation of students in vocational education. While previous studies demonstrated positive intervention effects, to enhance research-to-practice in real-world settings, it is important to gain an understanding of the key elements or mechanisms that transmit these outcomes. Next, we provide a brief overview of the personal safety competencies to which the intervention program is targeted.

Nykänen et al., (2018) defined safety preparedness as 'young peoples' readiness to implement actions that support occupational safety, and their resilience to deal with barriers or problems related to occupational safety and safe working" (p. 46). Safety preparedness is a cognitive construct comprising safety self-efficacy and preparation for barriers for safe work. Safety-related self-efficacy refers to the degree of confidence in one's ability to perform safety-related activities successfully at workplace, such as acquiring instructions or guidelines at work in order to work safely. Preparation for barriers for safe work refers to abilities to anticipate potential barriers and utilize behavioral strategies to resolve the respective problematic situations. (Nykänen et al., 2018). The internal safety locus of control refers to perceived control over occupational morbidity and mortality (Jones \& Wuebker, 1985). Both Internal safety locus of control and safety-related selfefficacy focus on competence-related beliefs, but each from a different perspective. Safety locus of control refers to a general perception of personal control over work-related incidents. Self-efficacy in turn refers to one's perceived ability to effectively perform specific behavioral activities (see Nykänen et al., 2019). Safety motivation has been defined as "an individual's willingness to exert effort to enact safety behaviors and the valence associated with those behaviors" (Neal \& Griffin, 2006. p. 947). Risk attitudes have been defined as the extent to which participants view occupational safety-related risk-taking at the workplace as appropriate (see Nykänen et al., 2018). 


\section{Current Study}

This study expands previous research by exploring associations between the core components of the Attitude to Work intervention and the targeted outcomes. Safety skills training and safety inoculation training utilize two learning mechanisms: mastery experiences of safety promotion activities and vicarious learning through social modeling of other students' performance during safety training activities. Bandura (1997) has highlighted that these two learning mechanisms are important sources of self-efficacy. Also, safety skills training guides students to identify the negative outcomes (e.g., work-related injuries and illness) of unsafe behavior at work and positive outcomes (e.g., avoiding injuries and illness) of preventive actions. Similarly, safety inoculation training stresses the importance of employee's own positive attitudes and actions in terms of overcoming barriers for safe work. According to expectancy value (Eccles \& Wigfield, 2002), the relative value that individuals place on certain activities and the perceived cost of certain actions are important determinants of attitudinal change. Thus, delivering safety skills training and safety inoculation training with high fidelity may contribute to positive, attitudinal outcomes of the program. Safety skills training and safety inoculation training activities also focus on perceptions of personal control over workrelated injuries and illness. Reorienting control beliefs may have an impact on internal safety locus of control (see Huang \& Ford, 2011). Previous research (Nykänen et al., 2019) indicates that modifying competencerelated beliefs contributed to the intervention effect on safety motivation. This may be reflected in how safety skills and safety inoculation relate to the motivational impact of the intervention. Furthermore, earlier studies (Hedlund et al., 2016; Rodrigues et al., 2018; Burke et al., 2006; Williams et al., 2010) indicate that active participation on the part of the learners is important in terms of the impact of safety training on motivational, attitudinal, and safety knowledge outcomes. Therefore, utilizing active learning methods in safety training programs may play an important role in achieving positive, intervention outcomes. Finally, earlier studies indicate that a supportive learning environment plays an important role in student motivation, engagement and learning (see Shernoff et al., 2017). Bandura (1997) also stresses the role of emotional arousal in predicting self-efficacy beliefs. Thus, it is expected that fostering a positive atmosphere contributes to intervention impacts.

Based on the earlier studies presented above, it can be expected that implementing the Attitude to Work core components with fidelity contributes to positive, intervention outcomes. However, a more detailed analysis of the relationships between the different components and the different outcome variables is needed (Abry et al., 2017). It is possible that a single core component may play a central role for one outcome variable while its role may be less important for others. The goal of this study was to acquire knowledge for future refinement, adaptation and scale-out of the Attitude to Work program to help prevent injuries and illnesses among younger workers.

\section{Method}

This study uses data collected during a school-based cluster randomized controlled trial (RCT), conducted in 2015 in eight Finnish upper secondary-level vocational schools. Information about how the trial was conducted and the efficacy of the Attitude to Work program has been reported in previously published studies (Nykänen et al., 2018, 2019). In the current study, we explored the associations between the core components and student outcome variables (safety preparedness, internal safety locus of control, risk attitudes, and safety motivation) to identify the active ingredients of the intervention. Figure 1 illustrates the associations between the concepts explored in the study.

\section{Study Participants}

In Finland, upper secondary vocational education concerns learning practical and work-specific skills in various occupational fields. The upper secondary vocational qualification takes approximately 3 years to complete. Students with this qualification are considered to have the basic vocational competence required for working life. Overall, 464 students from eight vocational schools participated in the previous RCT (Nykänen et al., 2018). The students were clustered in 44 student groups, the sizes of which ranged from 6 to 22 in the intervention condition $(M=13.6, S D=5.2)$ and from 6 to 26 in the control condition $(M=12.7, S D=5.0)$. The students in the control group did not participate in the program and did not provide study data for the analyses regarding association between intervention core components and targeted outcomes. Thus, the analyses presented here include only intervention condition participants $(n=229$ students clustered in 22 groups, each with one teacher).

\section{Measures}

Implementation Fidelity In our study, we focused on student perceptions of the implementation fidelity of the hypothesized 
Intervention core components

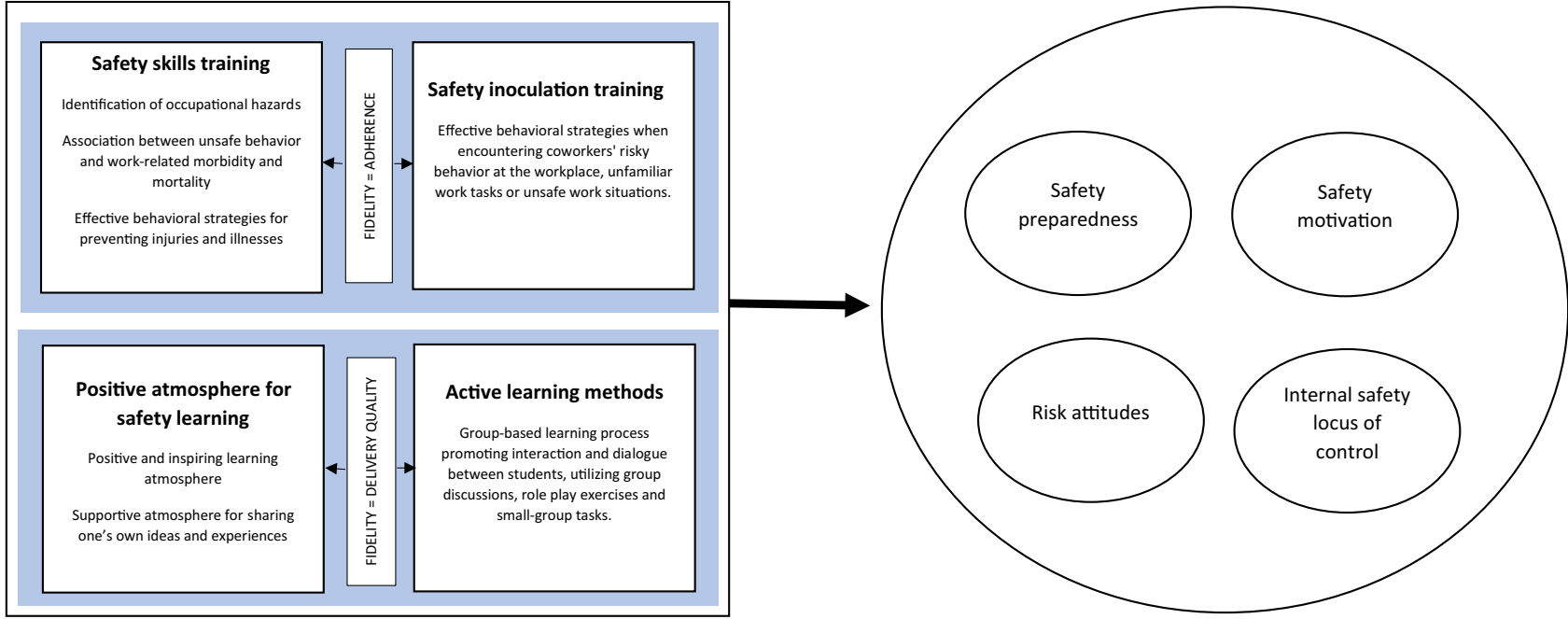

Fig. 1 A representation of intervention core components (on the left side of the figure) associated with measured outcomes (right). Fidelity of implementation (on the left) is defined as adherence to the educational content and quality of intervention delivery. When delivered with fidelity, the intervention core components illustrated in the figure are hypothesized to be the main contributors to the outcomes of interest

Safety Preparedness The safety preparedness measure is a nine-item measure of safety self-efficacy and safety inoculation. Six, 5-point ( $1=$ very poorly; $5=$ very well) self-efficacy items addressed safety-related activities at the workplace (example item: "acquiring instructions or guidelines at work in order to work safely"). Three, 5-point inoculation items ( $1=$ very few; $5=$ many) measured the extent to which participants had ideas or plans for situations in which they may encounter various safety-related problems in the workplace (example item: 'Co-workers' attitudes and behavior are harmful to occupational safety"). A more detailed description of the safety preparedness scale is provided in Nykänen et al. (2018). The internal consistency reliability $(\alpha)$ at baseline was 0.84 and at follow-up was 0.87 .

Internal Safety Locus of Control Internal safety locus of control was measured using a three, 5 -point $(1=$ strongly disagree; 5 = strongly agree) items (example item: "People can avoid injury if they are careful and aware of potential dangers") adapted from a measure by Mazaheri et al. (2012), $\alpha=0.67$ at time 1 and $\alpha=0.63$ at time 2 .

Safety Motivation In our study we used a 5-point ( $1=$ strongly disagree; $5=$ strongly agree) scale developed previously by Neal et al. (2000), consisted of three items (example item: "I feel that it is important to maintain safety at all times"), $\alpha=0.86$ at baseline and at follow-up $\alpha=0.84$. 
Risk-Taking Attitude The measure used in the current study measure was adapted from the general safety attitude scale developed by Henning et al. (2009). The five-point scale ( $1=$ strongly disagree; $5=$ strongly agree) included three items (example item: "Sometimes it is necessary to take risks to get a job done") that measured the extent to which participants viewed occupational safety-related risk-taking as appropriate at the workplace. At both baseline and follow-up, $\alpha=0.81$ for the risk attitude measure.

\section{Procedure}

Student participants (229 students in 22 groups) participated in the Attitude to Work intervention program implemented during the 2015 school year. Each school received a two-day teacher training workshop and the intervention program was implemented at school by the teachers within approximately 2 weeks of student baseline measurements. The intervention program consisted of 2 days of training and lasted $12 \mathrm{~h}$ in total. The students completed the follow-up questionnaires immediately after completing the Attitude to Work program.

\section{Analyses}

We explored the correlations, means, and standard deviations of the study variables using individual-level data. To describe the magnitude of intervention effects, we calculated effect sizes for between-group (intervention vs. control condition) differences regarding mean pre-post changes using the $d_{\mathrm{ppc} 2}$ formula by Morris (2008) which represent the standardized mean difference between intervention and control group. Effect size calculation is based on earlier intervention efficacy evaluations (Nykänen et al., 2018, 2019). Group-level variables were the focus of the analyses. Previous studies have described that student ratings can be aggregated at the studentgroup level to yield a measure of the "shared perception of the environment" (Lüdtke et al., 2009). Aggregated student group average variables refer to the shared perceptions of the group as a whole. In our study, we aggregated student-level responses as to their perceptions of teachers' implementation of intervention core component variables to form collective, group-level variables. Before aggregating these data, we calculated intra cluster correlations (ICC1 and ICC2) and within-group agreement statistics $\left(\mathrm{RWG}_{\mathrm{j}}\right)$ for all the grouplevel variables to justify the use of aggregated variables in the models.

We conducted confirmatory factor analysis using Mplus 7.4 software (Muthén and Muthén, Los Angeles, CA) to examine the proposed four-factor scale. We assessed model fit using the chi-square index $\left(\chi_{2}\right)$, the Tucker-Lewis index (TLI), the standardized root-mean-square residual (SRMR), the comparative fit index (CFI), and the root mean square error of approximation (RMSEA). Good model fit was evaluated using the following benchmarks: root-mean-square error of approximation (RMSEA $\leq 0.06$ ), standard root-meansquare residual (SRMR $<0.08$ ), and comparative fit index (CFI $\geq 0.95$; Hu \& Bentler, 1999). To test the hypothesis that the intervention's core components had positive associations with the intervention's outcomes, we estimated 16 separate models using generalized linear mixed models (GLMM) in SPSS version 25. The potential effect of clustering due to student groups was taken into account by using a random intercept model. Each model included a different core component and intervention outcome. All models were adjusted for baseline outcome values.

\section{Results}

\section{Descriptive Statistics}

Table 1 shows the means and the correlations of the study variables using an individual level of analysis. Standardized effect sizes were 0.33 for safety preparedness, 0.22 for internal safety locus of control, 0.17 for safety motivation, and 0.22 for risk attitudes indicating small intervention effects. The group-level properties regarding ICC(1) and ICC(2) of the intervention's core components across the intervention condition student groups $(n=22)$ are presented in Table 1 . The mean $\mathrm{RWg}_{(\mathrm{j})}$ values were as follows: for safety skills training, 0.79 (ranged between 0.67 and 0.92 ); for safety inoculation training 0.76 (ranged between 0.59 and 0.88 ); for positive learning atmosphere 0.74 (ranged between 0.46 and 0.92 ); and for active learning techniques 0.72 (ranged between 0.49 and 0.91 ). Thus, mean $\mathrm{RWg}_{(\mathrm{j})}$ values $(>0.70)$ indicated generally accepted agreement levels in core component measures.

\section{Association Between Core Components and Intervention Outcomes}

Confirmatory factor analysis regarding intervention fidelity measurement indicated an acceptable four-factor model fit $\left(\left(\chi^{2}(98)=202.294, p<0.001, \mathrm{CFI}=0.940, \mathrm{TLI}=0.926\right.\right.$, SRMR $=0.049$, RMSEA $=0.067$ (95\% confidence interval $=0.054-0.080)$ ). All the factor loadings of the manifest indicators were significant $(p<0.001)$ and were between 0.46 and 0.87 . The standardized factor loadings of the intervention fidelity measurement are presented in the Online Resource A.

Table 2 presents the results of 16 multilevel models exploring the association between core components and intervention outcomes. The results of our analyses demonstrated positive associations between the intervention's core components and the student outcomes. Safety skills 


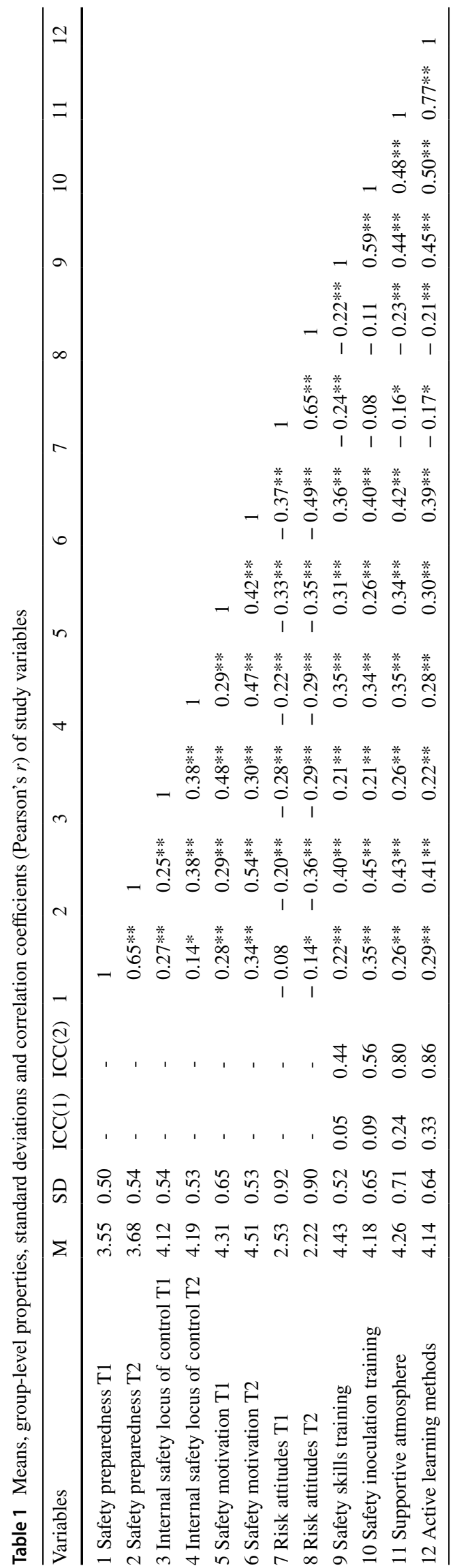

training had statistically significant associations with safety preparedness $(b=0.52, p<0.01)$, internal safety locus of control $(b=0.65, p<0.01)$, and safety motivation $(b=0.37, p<0.05)$. Safety inoculation training had similarly statistically significant associations with safety preparedness ( $b=0.28, p<0.05)$ and internal safety locus of control ( $b=0.30, p<0.05)$ but not with safety motivation. We also found that a positive learning atmosphere had a statistically significant relationship with safety preparedness $(b=0.17$, $p<0.05)$ and safety motivation $(b=0.20, p<0.05)$. Finally, active learning techniques had a positive association only with safety motivation $(b=0.18, p<0.05)$. Contrary to our expectations, we found no statistically significant relationship between any of the core components and risk-taking attitudes.

\section{Discussion}

Given that schools have limited resources for implementing new prevention programs, ready to-use, evidence-based programs are important for increasing the adoption and implementation of OSH training and education in schoolbased contexts (Guerin et al., 2019). Our study highlights that the focus on adherence to the educational content is not enough, but attention should also be paid to the manner which the evidence-based safety training programs in schools are implemented. Overall, our results demonstrated that the fidelity measurement of adherence related to the acquisition of safety skills training was the strongest active ingredient in Attitude to Work safety training program. Adherence related to safety inoculation training had similar associations regarding competence-related beliefs, but the results showed no significant relationship with safety motivation. This result may be related to the limited work experience of young people. A lack of previous personal experiences on safety related barriers may affect an individual's perception of the importance of advance preparation for them. The positive connection of safety skills training and safety inoculation training to competence-related beliefs can be interpreted through the lens of social-cognitive theory. Students identify and practice behavioral strategies to prevent work-related morbidity and mortality and to overcome barriers for safe work. During these training activities, students are given the opportunity to notice the development of their safety skills (mastery experiences) and can observe peers as they engage in learning process (vicarious learning). Students are also guided toward identifying controllable causes of workrelated incidents which in turn can have a positive impact on perceived control over safety.

The study results suggest that fostering a positive and supportive learning atmosphere contributes to motivational impact of safety training activities. Moreover, we found that utilizing active learning techniques were related to 
Table 2 Summary of 16 multilevel models exploring the association between intervention core components and student outcomes

\begin{tabular}{|c|c|c|c|c|c|c|c|c|}
\hline & \multicolumn{2}{|c|}{ Safety preparedness } & \multicolumn{2}{|c|}{$\begin{array}{l}\text { Internal safety locus of } \\
\text { control }\end{array}$} & \multicolumn{2}{|c|}{ Risk-taking attitudes } & \multicolumn{2}{|c|}{ Safety motivation } \\
\hline & Estimate & $95 \% \mathrm{CI}$ & Estimate & $95 \% \mathrm{CI}$ & Estimate & $95 \% \mathrm{CI}$ & Estimate & $95 \% \mathrm{CI}$ \\
\hline Group-level safety skills training & $0.52 * *$ & $0.27,0.77$ & $0.65 * *$ & $0.34,0.97$ & -0.26 & $-0.82,0.29$ & $0.37 *$ & $0.06,0.69$ \\
\hline Group-level safety inoculation training & $0.28 *$ & $0.06,0.50$ & $0.30 *$ & $0.03,0.57$ & 0.02 & $-0.39,0.44$ & 0.18 & $-0.07,0.44$ \\
\hline Group-level positive learning atmosphere & $0.17 *$ & $0.02,0.31$ & 0.14 & $-0.04,0.33$ & -0.11 & $-0.39,0.15$ & $0.20 *$ & $0.03,0.36$ \\
\hline Group-level active learning techniques & 0.13 & $-0.01,0.28$ & 0.11 & $-0.07,0.30$ & -0.17 & $-0.44,0.08$ & $0.18 *$ & $0.03,0.34$ \\
\hline
\end{tabular}

Student group was included as a random effect to account for clustering in all study models. All models were adjusted for baseline outcome value

safety motivation. This result is comparable with a study by Hedlund et al. (2016) that demonstrated that interventions where the participants had a high degree of participation led to increased safety motivation. In our study, quality of delivery in terms of utilizing active learning methods demonstrated no associations with other outcome variables. These results can be interpreted as that the benefits of active learning methods in school-based safety training may relate to motivational outcomes, but for competence-related beliefs, such as internal safety locus of control and safety preparedness, the delivery of educational content with high fidelity perhaps may play a more central role.

A previous RCT (Nykänen et al., 2018) indicated that the risk attitudes of students who participated in the Attitude to Work training program decreased more than those of the students in the control condition. However, we found no statically significant associations between intervention core components and risk attitudes. This suggests that the intervention effect on attitudes was influenced by an unmeasured factor not considered in the prespecified intervention model (see Rojas-Andrade \& Bahamondes, 2019). According to Damschroder et al., (2009), intervention implementation is "a social process that is intertwined with the context in which it takes place." The safety training program is based on a dialogue between students and therefore may have activated a process of constructing more positive social norms among student participants. The injunctive safety norm concept provides one perspective on this matter. Injunctive safety norms refer to the extent to which individuals perceive others' approval and expectations of safety-related behavior (Fugas et al., 2011). Previous studies have demonstrated that young workers' risk-taking orientation is influenced by peer workers behaviors (Westaby \& Lowe, 2005). Furthermore, Reid and Aiken (2013) found that changes in injunctive norms and normative feedback had a positive impact on attitude toward sun protective behaviors. Research from Pek et al. (2017) also provides empirical support for how injunctive safety norms may be associated with young workers' job-related risk-taking behaviors. Hence, it possible that the group-learning process and safety related communication between students had an impact on perceived injunctive safety norms which in turn was the key factor in facilitating attitudinal changes. However, our fidelity measures did not assess the perceptions of injunctive safety norms. Further research is needed to explore this topic, including by measuring injunctive safety norms before and after safety training based on the peer learning process.

\section{Study Limitations}

One study limitation is that we had no observational data on the implementation process or teacher reports on the delivery of the program core components. Also, our analyses indicate low group mean reliability for safety skills training and safety inoculation training measures. The relatively small student group sizes may have had an impact on the ICC values in our study data (Bliese, 2000). Finally, standardized effect sizes indicate only small intervention effects. However, even small intervention effects can be practically important if the intervention program is cost efficient and scalable (see Bakker et al., 2019). School-based interventions have the potential to reach a large number of young people entering working life and Attitude to Work program offers a readyto-use, free, and publicly available training method for preparing future workers for safe and healthy employment.

\section{Future Research in Prevention Science}

The current study advances prevention science by identifying the active ingredients of the evidence-based, Attitude to Work intervention, implemented in Finnish vocational school settings, that helps protect young workers from work-related morbidity and mortality.

Future research should explore interactions between the intervention active ingredients. In terms of the Attitude to Work training program, a positive learning atmosphere may be more 
supportive of active learning during small group assignments and role-play exercises. Furthermore, to better understand the mechanisms of change school-based interventions, it is important to consider the interplay between the intervention process and contextual factors. The delivery, with fidelity, of intervention core components may interact with the social context and produce unplanned intervention effects. This perspective is in line with earlier research (Kelly, 2012) that found that implementation evaluation should increase understanding of relevant contextual processes that play important role in intervention effectiveness. Exploring the interaction between the intervention program and the context may require a more holistic approach, such as by utilizing a mixed-methods design, to obtain a fuller picture of implementation processes. Finally, a discussion of balancing fidelity and adaptation to meet the local needs and constraints of program providers and recipients (Allen et al., 2018; Stirman et al., 2019) is a topic of scholarly interest in prevention science (see e.g., Bopp et al., 2013; Castro et al., 2004). Research should be focused on how the Attitude to Work intervention may be modified to fit different school, cultural, and other contexts and stakeholder needs to ensure that the future workforce is equipped with the knowledge, skills, and abilities they need to stay safe and healthy on the job.

Supplementary Information The online version contains supplementary material available at https://doi.org/10.1007/s11121-021-01209-8.

Funding This article is based on research supported by the Academy of Finland (\#293445) and the Finnish Work Environment Fund (\#113086).

\section{Compliance with Ethical Standards}

Disclaimer The findings and conclusions in this report are those of the authors and do not necessarily represent the views of the National Institute for Occupational Safety and Health, Centers for Disease Control and Prevention.

Conflicts of Interest The authors declare that they have no conflict of interest.

Ethical Approval All procedures performed in studies involving human participants were in accordance with the ethical standards of the ethics committee at the Finnish Institute of Occupational Health (\#1/2015) and with the 1964 Helsinki declaration and its later amendments or comparable ethical standards.

Informed Consent Informed consent was obtained from all individual participants included in the study.

Open Access This article is licensed under a Creative Commons Attribution 4.0 International License, which permits use, sharing, adaptation, distribution and reproduction in any medium or format, as long as you give appropriate credit to the original author(s) and the source, provide a link to the Creative Commons licence, and indicate if changes were made. The images or other third party material in this article are included in the article's Creative Commons licence, unless indicated otherwise in a credit line to the material. If material is not included in the article's Creative Commons licence and your intended use is not permitted by statutory regulation or exceeds the permitted use, you will need to obtain permission directly from the copyright holder. To view a copy of this licence, visit http://creativecommons.org/licenses/by/4.0/.

\section{References}

Abry, T., Rimm-Kaufman, S. E., \& Curby, T. W. (2017). Are all program elements created equal? Relations between specific social and emotional learning components and teacher-student classroom interaction quality. Prevention Science, 18, 193-203.

Abry, T., Hulleman, C. S., \& Rimm-Kaufman, S. E. (2015). Using indices of fidelity to intervention core components to identify program active ingredients. American Journal of Evaluation, 36, 320-338.

Allen, J. D., Linnan, L. A., Emmons, K. M., Brownson, R., Colditz, G., \& Proctor, E. (2018). Fidelity and its relationship to implementation effectiveness, adaptation, and dissemination. In R.C. Brownson, G.A. Colditz, E.K. Proctor, Dissemination and Implementation Research in Health: Translating Science to Practice. (pp. 281-304). 2nd Edition. Oxford University Press, New York, NY.

Backer, T.E. (2001). Finding the Balance: Program Fidelity and Adaptation in Substance Abuse Prevention: A State-of-the-Art Review. Rockville, MD: Center for Substance Abuse Prevention, Substance Abuse and Mental Health Services Administration. 1-82.

Bandura, A. (1997). Self-efficacy: The exercise of control. NY: W.H. Freeman and company.

Bakker, A., Cai, J., English, L., Kaiser, G., Mesa, V \& Van Doore, W.(2019). Beyond small, medium, or large: Points of consideration when interpreting effect sizes. Educational Studies in Mathematics, 102, 1-8.

Bast, L., Andersen, A., Ersbøll, A., \& Due, P. (2019). Implementation fidelity and adolescent smoking: The X:IT study-A school randomized smoking prevention trial. Evaluation and Program Planning, 72, 24-32.

Berkel, C., Mauricio, A. M., Schoenfelder, E., \& Sandler, I. N. (2011). Putting the pieces together: An integrated model of program implementation. Prevention Science, 12, 23-33.

Bliese, P.D. (2000). Within-group agreement, non-independence, and reliability. In Kline, K.J., Kozlowski, S.W. Multilevel Theory, Research, and Methods in Organizations. (pp. 349-381), JosseyBass, San Francisco,

Bopp, M., Saunders, R. P., \& Lattimore, D. (2013). The tug-of-war: fidelity versus adaptation throughout the health promotion program life cycle. The Journal of Primary Prevention, 34, 193-207.

Boini, S., Colin, R., \& Grzebyk, M. (2017). Effect of occupational safety and health education received during schooling on the incidence of workplace injuries in the first 2 years of occupational life: A prospective study. BMJ Open, 7.

Breslin, F., \& Smith, P. (2006). Age-related differences in work injuries: A multivariate, population-based study. American Journal of Industrial Medicine, 48, 50-56.

Burke, M. J., Sarpy, S. A., Smith-Crowe, K., Chan-Serafin, S., Salvador, R. O., \& Islam, G. (2006). Relative effectiveness of worker safety and health training methods. American Journal of Public Health, 96, 315-324.

Carroll, C. (2020). Fidelity. In P. Nilsen \& S.A. Birken, Handbook on Implementation Science (pp. 291-316). Edward Elgar Publishing. Massachusetts. USA.

Castro, F. G., Barrera, M., \& Martinez, C. R. (2004). The cultural adaptation of prevention interventions: Resolving tensions between fidelity and fit. Prevention Science, 5, 41-45. 
Collins, L., Murphy, S., Nair, V., \& Strecher, V. (2005). A strategy for optimizing and evaluating behavioral interventions. Annals of Behavioral Medicine, 30, 65-73.

Damschroder, L. J., Aron, D. C., Keith, R. E., Kirsh, S. R., Alexander, J. A., \& Lowery, J. C. (2009). Fostering implementation of health services research findings into practice: A consolidated framework for advancing implementation science. Implementation Science, 4, 50.

Dane, A. V., \& Schneider, B. (1998). Program integrity in primary and early secondary prevention: Are implementation effects out of control? Clinical Psychology Review, 18, 23-24.

Dugan, A. G., \& Punnett, L. (2017). Dissemination and implementation research for occupational safety and health. Occupational Health Science, 1, 29-45.

Dusenbury, L., Brannigan, R., Falco, M., \& Hansen, W. (2003). A review of research on fidelity of implementation: Implications for drug abuse prevention in school settings. Health Education Research, 18, 237-256.

Dunst, C. J., Trivette, C. M., \& Raab, M. (2013). An implementation science framework for conceptualizing and operationalizing fidelity in early childhood intervention studies. Journal of Early Intervention, 35, 85-101.

Durlak, J. A., \& DuPre, E. P. (2008). Implementation matters: A review of research on the influence of implementation on program outcomes and the factors affecting implementation. American Journal of Community Psychology, 41, 327-350.

Eccles, J. S., \& Wigfield, A. (2002). Motivational beliefs, values, and goals. Annual Review of Psychology, 53, 109-132.

Espada, J. P., Griffin, K. W., Pereira, J. R., Orgilés, M., \& GarcíaFernández, J. M. (2012). Component analysis of a school-based substance use prevention program in Spain: Contributions of problem solving and social skills training content. Prevention Science, 13, 86-95.

Fugas, C., Melia, J., \& Silva, S. (2011). The "Is" and the "Ought": How do perceived social norms influence safety behaviors at work? Journal of Occupational Health Psychology, 16, 67-79.

Fixsen, D. L., Blase, K. A., Naoom, S. F., \& Wallace, F. (2009). Core implementation components. Research on social work practice, 19, 531-540.

Gearing, R. E., El-Bassel, N., Ghesquiere, A., Baldwin, S., Gillies, J., \& Ngeow, E. (2011). Major ingredients of fidelity: A review and scientific guide to improving quality of intervention research implementation. Clinical Psychology Review, 31, 79-88.

Guerin, R. J., Castillo, D., Hendricks, K., Piacentino, J., Howard, J., \& Okun, A. H. (2020). Preparing young workers for a lifetime of safe and healthy employment. American Journal of Public Health, 110, 69-71.

Guerin, R. J., Okun, A. H., Barile, J. P., Emshoff, J. G., Ediger, M. D., \& Baker, D. S. (2019). Preparing teens to stay safe and healthy on the job: A multilevel evaluation of the Talking Safety curriculum for middle schools and high schools. Prevention Science., 20, 510-520.

Hanvold, T., Kines, P., Nykänen, P., Thomée, S., Holte, K., Vuori, J., \& Veiersted, K. (2019). Occupational safety and health among young workers in the Nordic countries: A systematic literature review. Safety and Health at Work, 10, 3-20.

Hedlund, A., Gummesson, K., Rydell, A., \& Andersson, I. (2016). Safety motivation at work: Evaluation of changes from six interventions. Safety Science, 82, 155-163.

Henning, J., Stufft, C., Payne, S., Bergman, M., Mannan, M., \& Keren, N. (2009). The influence of individual differences on organizational safety attitudes. Safety Science, 47, 337-345.

Hu, L., \& Bentler, P. M. (1999). Cutoff criteria for fit indexes in covariance structure analysis: Conventional criteria versus new alternatives. Structural Equation Modeling, 6, 1-55.
Huang, J., \& Ford, K. (2011). Driving locus of control and driving behaviors: Inducing change through driver training. Transportation Research Part F: Traffic Psychology and Behaviour, 15, 358-368.

Irwin, M., \& Supplee, L. H. (2012). Directions in implementation research methods for behavioral and social science. The journal of behavioral health services \& research, 39, 339-342.

Jones, J., \& Wuebker, L. (1985). Development and validation of the safety locus of control scale. Perceptual and Motor Skills, 61, 151-161.

Kelly, B. (2012). Implementation science for psychology in education. In B. Kelly \& D. Perkins (Eds.), Handbook of implementation science for psychology in education (pp. 3-13). New York: Cambridge University Press.

Koehoorn, M., Breslin, F. C., \& Xu, F. (2008). Investigating the longer-term health consequences of work-related injuries among youth. Journal of Adolescent Health, 43, 466-473.

Leaper, C. (2011). More similarities than differences in contemporary theories of social development? A plea for theory bridging. Advances in Child Development and Behavior, 40, 337-378.

Lüdtke, O., Robitzsch, A., Trautwein, U., \& Kunter, M. (2009). Assessing the impact of learning environments: How to use student ratings of classroom or school characteristics in multilevel modeling. Contemporary Educational Psychology, 34, 120-131.

Mowbray, C., Holter, M., Teague, G., \& Bybee, D. (2003). Fidelity criteria: Development, measurement, and validation. American Journal of Evaluation, 24, 315-340.

Neal, A., \& Griffin, M. (2006). Study of the lagged relationships among safety climate, safety motivation, safety behavior, and accidents at the individual and group levels. Journal of Applied Psychology, 91, 946-953.

Neal, A., Griffin, M. A., \& Hart, P. M. (2000). The impact of organizational climate on safety climate and individual behavior. Safety Science, 34, 99-109.

Nykänen, M., Salmela-Aro, K., Tolvanen, A., \& Vuori, J. (2019). Safety self-efficacy and internal locus of control as mediators of safety motivation-Randomized controlled trial (RCT) study. Safety Science, 117, 330-338.

Nykänen, M., Sund, R., \& Vuori, J. (2018). Enhancing safety competencies of young adults. A randomized field trial (RCT). Journal of Safety Research, 67, 45-56.

Mazaheri, M., Hidarnia, A \& Ghofranipour, G. (2012). Safety education and control: A tool to measure the safety locus of control. Journal of Education and Health Promotion, 1.

Morris, S. B. (2008). Estimating effect sizes from pretest-posttestcontrol group designs. Organizational Research Methods, 11, 364-386.

Pek, S., Turner, N., Tucker, S., Kelloway, E. K., \& Morrish, J. (2017). Injunctive safety norms, young worker risk-taking behaviors, and workplace injuries. Accident Analysis \& Prevention, 106, 202-210.

Reid, A., \& Aiken, L. (2013). Correcting injunctive norm misperceptions motivates behavior change: A randomized controlled sun protection intervention. Health Psychology, 32, 551-560.

Rohrbach, L. A., Grana, R., Sussman, S., \& Valente, T. W. (2006). Type II translation: Transporting prevention interventions from research to real-world settings. Evaluation \& Health Care Professionals, 29, 302-333.

Rodrigues, M., Vale, C., \& Silva, M. (2018). Effects of an occupational safety programme: A comparative study between different training methods involving secondary and vocational school students. Safety Science, 109, 353-360.

Rojas-Andrade, R., \& Bahamondes, L. (2019). Is Implementation fidelity important? A systematic review on school-based 
mental health programs. Contemporary School Psychology, 23, 339-350.

Rotter, J. B. (1982). The development and applications of social learning theory. New York: Praeger.

Schoenwald, S. K., Garland, A. F., Chapman, J. E., Frazier, S. L., Sheidow, A. J., \& Southam-Gerow, M. A. (2011). Toward the effective and efficient measurement of implementation fidelity. Administration and Policy in Mental Health and Mental Health Services Research, 38, 32-43.

Shernoff, D. J., Ruzek, E. A., \& Sinha, S. (2017). The influence of the high school classroom environment on learning as mediated by student engagement. School Psychology International, 38, 201-218.

Schulte, P. A., Cunningham, T. R., Nickels, L., Felknor, S., Guerin, R., Blosser, F., \& Forrester, C. (2017). Translation research in occupational safety and health: A proposed framework. American Journal of Industrial Medicine, 60, 1011-1022.
Stirman, S. W., Baumann, A. A., \& Miller, C. J. (2019). The FRAME: an expanded framework for reporting adaptations and modifications to evidence-based interventions. Implementation Science, 14, 1-10.

Vuori, J., Price, R. H., Mutanen, P., \& Malmberg-Heimonen, I. (2005). Effective group training techniques in job search training. Journal of Occupational Health Psychology, 10, 261-275.

Westaby, J. D., \& Lowe, J. K. (2005). Risk taking orientation and injury among youth workers: Examining the social influence of supervisors, coworkers, and parents. Journal of Applied Psychology, 90, 1297-1305.

Williams, Q., Jr., Ochsner, M., Marshall, E., Kimmel, L., \& Martino, C. (2010). The impact of a peer-led participatory health and safety training program for Latino day laborers in construction. Journal of safety research, 41, 253-261. 